
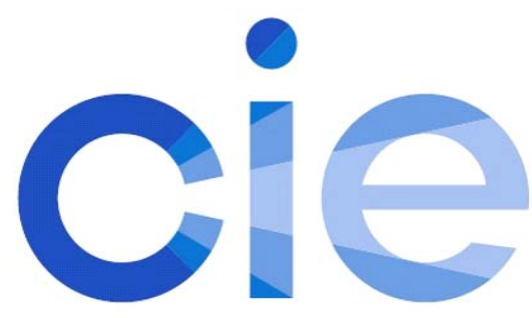

PO042

\title{
MEASUREMENTS OF INTRAOCULAR STRAYLIGHT, VISUAL SENSITIVITY, AND DISCOMFORT GLARE FOR YOUNG AND ELDERLY OBSERVERS
}

Yuki Tachikawa et al.

DOI 10.25039/x46.2019.PO042

from

CIE x046:2019

Proceedings

of the

29th CIE SESSION

Washington D.C., USA, June 14 - 22, 2019

(DOI 10.25039/x46.2019)

The paper has been presented at the 29th CIE Session, Washington D.C., USA, June 14-22, 2019. It has not been peer-reviewed by CIE.

(c) CIE 2019

All rights reserved. Unless otherwise specified, no part of this publication may be reproduced or utilized in any form or by any means, electronic or mechanical, including photocopying and microfilm, without permission in writing from CIE Central Bureau at the address below. Any mention of organizations or products does not imply endorsement by the CIE.

This paper is made available open access for individual use. However, in all other cases all rights are reserved unless explicit permission is sought from and given by the CIE.

CIE Central Bureau

Babenbergerstrasse 9

A-1010 Vienna

Austria

Tel.: +4317143187

e-mail: ciecb@cie.co.at

www.cie.co.at 


\title{
MEASUREMENTS OF INTRAOCULAR STRAYLIGHT, VISUAL SENSITIVITY, AND DISCOMFORT GLARE FOR YOUNG AND ELDERLY OBSERVERS
}

\author{
Tachikawa, Y. ${ }^{1}$, Tsuchiya, N. ${ }^{1}$, Ishikawa, T. ${ }^{1}$, Ohnuma, K. ${ }^{2}$, Ayama, M. ${ }^{1}$ \\ ${ }^{1}$ Utsunomiya University, Tochigi, JAPAN \\ ${ }^{2}$ Chiba University, Chiba, JAPAN \\ mt186720@cc.utsunomiya-u.ac.jp
}

DOI 10.25039/x46.2019.PO042

\begin{abstract}
To reveal the mutual relation between the intraocular straylight, visual sensitivity, and discomfort glare for elderly and young observers, triad measurements were carried out using the same white LED source. Multiple regression analysis to estimate discomfort glare sensitivity using straylight parameter and visual sensitivity as explanatory variables was applied to the results of both groups. Correlation coefficient between the estimated and experimental values is 0.67 for elderly group, indicating that these two factors contribute to the subjective evaluation of discomfort glare for individual observers. On the other hand, estimation was not so good for young group, suggesting that other factors, e.g., psychological criterion difference of subjective rating, affect on individual differences of discomfort glare for young observers.
\end{abstract}

Keywords: Intraocular straylight, Visual sensitivity, Discomfort glare, White LED

\section{Introduction}

Disability glare is known as the light-veil by glare sources that disturbs a visibility of target caused by straylight in the eye. Factors that brought straylight in the eye are the forward scatter by the ocular media such as crystalline lens, the fundus reflection, and the penetration through sclera and iris [1, 2]. We have been studying to estimate the amount of straylight in the eye using psychophysical technique for the last few years to accumulate data for elderly and young observers $[3,4]$. Because we employed a veiling method, results of the step 1 of the straylight measurement reflect disability glare property of individual observers.

Discomfort glare is known as a dazzling of light that causes uncomfortable sensation [5]. A number of studies have been reported on discomfort glare using LED lighting in the past 10 years [6-11]. Let's say the the border luminance between acceptable and unacceptable discomfort glare, Lborder,glare, here. About the age dependency, the previous study by Kimura et al. [7] indicated no significant difference of $L$ border,glare between elderly and young observers for various color LEDs except the blue LED. They tried to explain the results by the decreases of crystalline-lens transparency and spectral brightness sensitivity of elderly observers in shortwavelength region. Their analysis was not fully successful, partly because the spectral brightness sensitivity functions employed in their study were not measured using their observers. Also, we consider not the brightness sensitivity, but the absolute visual sensitivity of individuals might relate to $L_{\text {border, glare. }}$

Few has been reported on the factors to contribute discomfort glare except the study by Bargary et al. [12]. Objective of this study is to reveal the relation between the straylight in the eye, visual sensitivity, and discomfort glare for elderly and young observers.

\section{Experiments}

\subsection{Estimation of intraocular straylight}

White, blue, green, and red LED lights were used as glare sources and set at 7 degree on the right-hand side from the line of sight. Illuminance at the eye, denoted as $E_{\text {glare, }}$ was set to 7 to 9 lux. A Gabor stimulus, of which carrier wave of spatial frequency is $2.2 \mathrm{cpd}$ and standard deviation of the envelope wave is $0.46 \mathrm{deg}$, was used as the visual target. 
Observer was asked to see the visual target by either left or right eye, while the other eye was covered by an eye-patch. The first step was to determine the threshold contrast of the Gabor stimulus with the illumination of one of the glare sources. In the second step, the same glare source was moved to the position perpendicular to the observer's line of sight and superimposed to the Gabor stimulus using the half mirror. Luminance to veil the Gabor stimulus determined in the first step, $L_{e q}$, was measured. Then the straylight parameter, $s$, indicated in the equation 1 below was calculated. Log $s$ is widely used as an index to the amount of straylight in the eye.

$$
\mathbf{s}=L_{e q} \cdot \theta^{2} / E_{\text {glare }}
$$

\subsection{Discomfort glare measurement}

The white LED used in the staylight experiment was used as a test light. It was set at the same position, 7 degree on the right-hand side from the line of sight. Several ND filters were combined to generate 7 levels of illuminance. Three ranges of illuminance, 0.01 to $2.4 \mathrm{Ix}, 0.01$ to $5 \mathrm{Ix}$, and 0.01 to $10 \mathrm{~lx}$, were prepared. Proper range for individual observer was determined in the preexperiment. Subjective evaluation was carried out using 9 evaluation words, "1:Noticeable, 3:Acceptable, 5:Just admissible, 7:Disturbing, 9:Unbearable". Measurement was done using observers' left eye.

\subsection{Visual sensitivity measurement}

To measure the detection threshold of individual observer using the same apparatus, the following experiment was carried out. The same white LED was used as the test stimulus and the ND filters were combined to generate 10 levels of luminance. One session began with 5 min dark adaptation, and the first stimulus was presented $0.5 \mathrm{sec}$. Observer was asked to answer "seen" or "not seen" by using 2AFC method. This was repeated 110 times in a session in a random order, including a dummy (no stimulus). Two to 4 sessions were carried out for each observer. Measurement was done using observers' left eye. Luminance value of $50 \%$ seen in the probability of seeing curve, $L_{t h}$, was defined as the threshold.

\subsection{Observers}

Eleven elderly in their 60's and 11 young in their 20's participated all experiments.

\section{Results}

\subsection{Results of each measurement}

In this study, only the results of the measurement using white LED as a glare source and observers' left eye are shown because discomfort glare and visual sensitivity were measured only under that condition. Change of log $s$ with the peak wavelength of the LED was reported by Tsuchiya et al. [4].

Figure 1 (a) shows the results of the first step in the veiling method. Vertical axis indicates peak luminance of the Gabor stimulus of foveal detection threshold with the light veil of the white

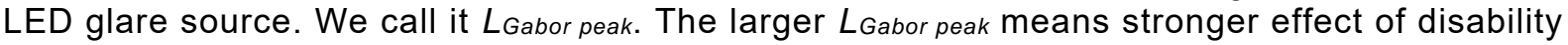
glare. As shown in the figures, $L_{\text {Gabor peak }}$ is significantly higher in elderly group than young group, indicating that visibility is more easily disturbed for the elderly than the young observers. Figure 1 (b) shows the results of straylight parameter plotted in a logarithmic scale. Vertical axis, log $s$, indicates the amount of intraocular straylight. Consistent with results of previous studies [2], elderly people show larger value of straylight parameter than young observers. This is considered due to the yellowing of crystalline lens that increase the forward scattering in ocular media. It is known that other factors affect the amount of intraocular stray light, such as fundus reflection, and the penetration through sclera and iris [2], while the forward scattering is considered to be the most effective cause. Our results in Figure 1 (b) is consistent with the view. It is worth noting that the correlation coefficients between $L_{\text {Gabor peak }}$ and log $s$ among individual observers for all observers is 0.77 indicating strong positive correlation. However, this also indicates that $\log s$ is not determined only by LGabor peak.

In the measurement of discomfort glare, rating score increases as the illuminance at the eye increases for all observers. Border illuminance at the eye, $E_{\text {border,glare, that crosses the scale of }}$ 
5 , just admissible, was derived by the fitting using sigmoid function for individual results. Figure 2 shows the results of elderly and young groups. Vertical axis indicates logarithmic value of the

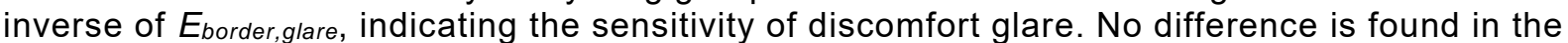
between the median of the two groups while the individual difference is larger in the elderly group.

Figure 3 shows the results of visual sensitivity. Inverse of the threshold luminance, $L_{t h}$, in logarithmic scale, that means the visual sensitivity, is plotted in the vertical axis. As shown in the figure, the young group shows higher sensitivity than the elderly group.

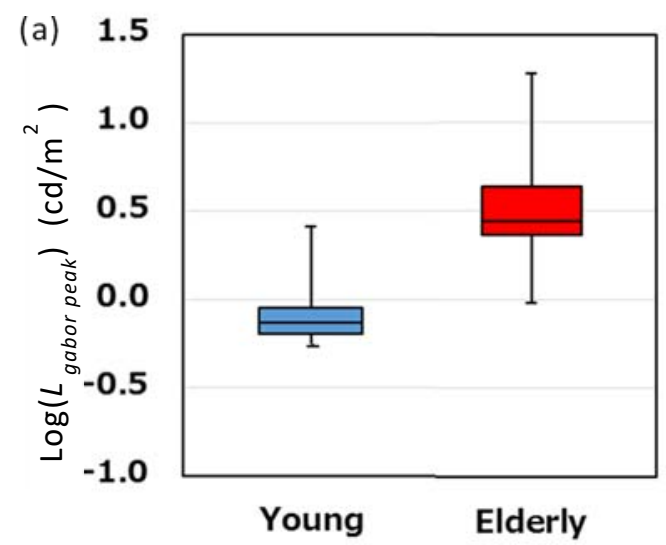

Observers

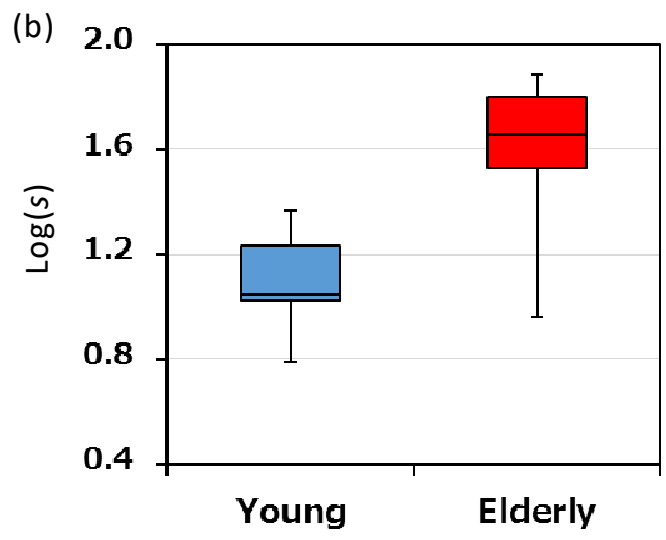

Observers

Figure 1 - Results of the intraocular straylight measurement. (a):Peak luminance of Gabor stimulus detected with glare source, (b):Straylight parameter

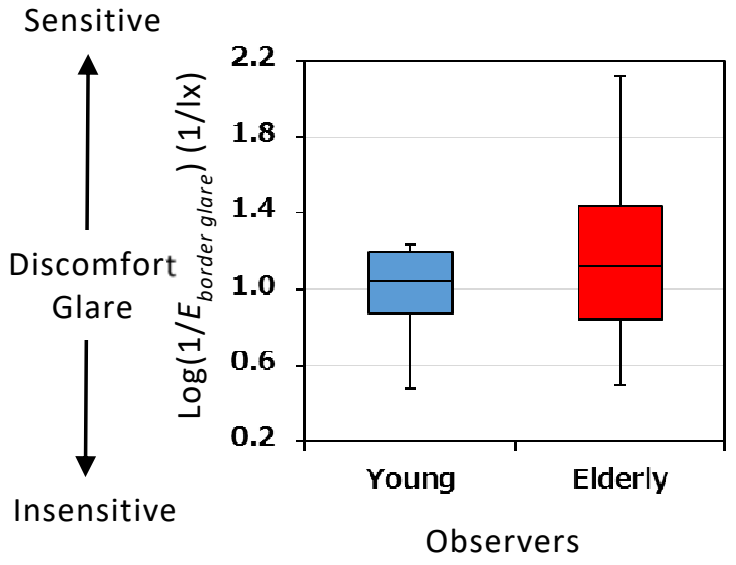

Figure 2 - Results of discomfort glare measurement

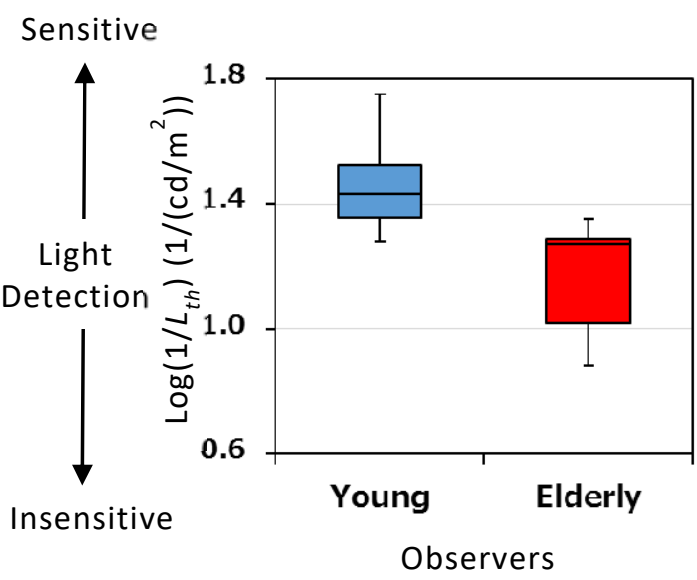

Figure 3 - Results of visual sensitivity

\subsection{Relation between intraocular straylight, visual sensitivity, and discomfort glare}

Figure 4 shows the relation between $\log s$ and discomfort glare sensitivity. There exist large individual differences in elderly group, but weak correlation is found in each of young and elderly groups that the larger the amount of straylight, the more sensitive to discomfort glare. Correlation coefficients for young and elderly group are 0.36 and 0.34 , respectively.

Figure 5 shows the relation between visual sensitivity and discomfort glare sensitivity. Here also large individual differences It is interesting that medium correlation is found in elderly group, while no. Correlation coefficients for young and elderly group are 0.36 and 0.34 , respectively. 


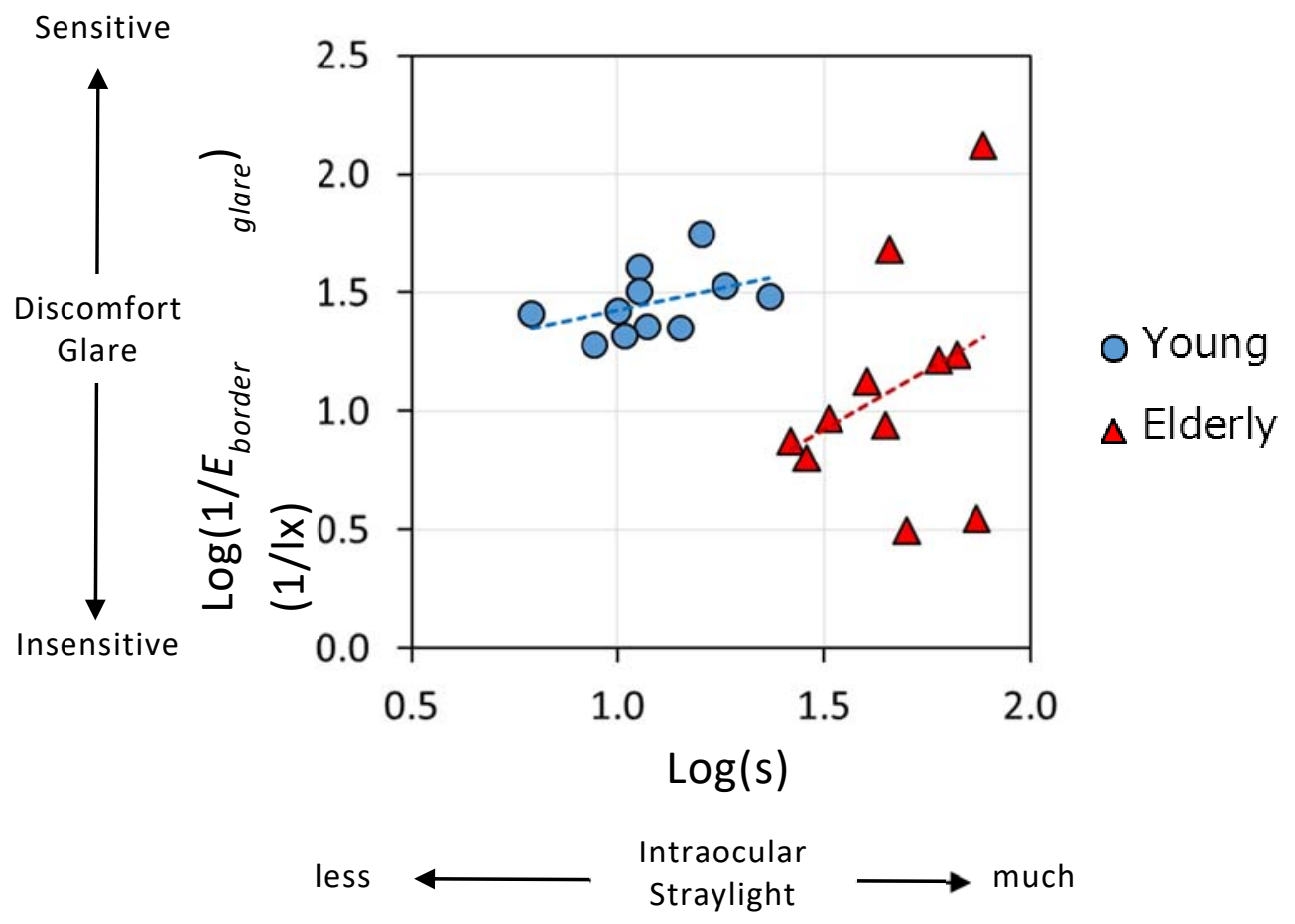

Figure 4 - Correlation between log s and the sensitivity of discomfort glare

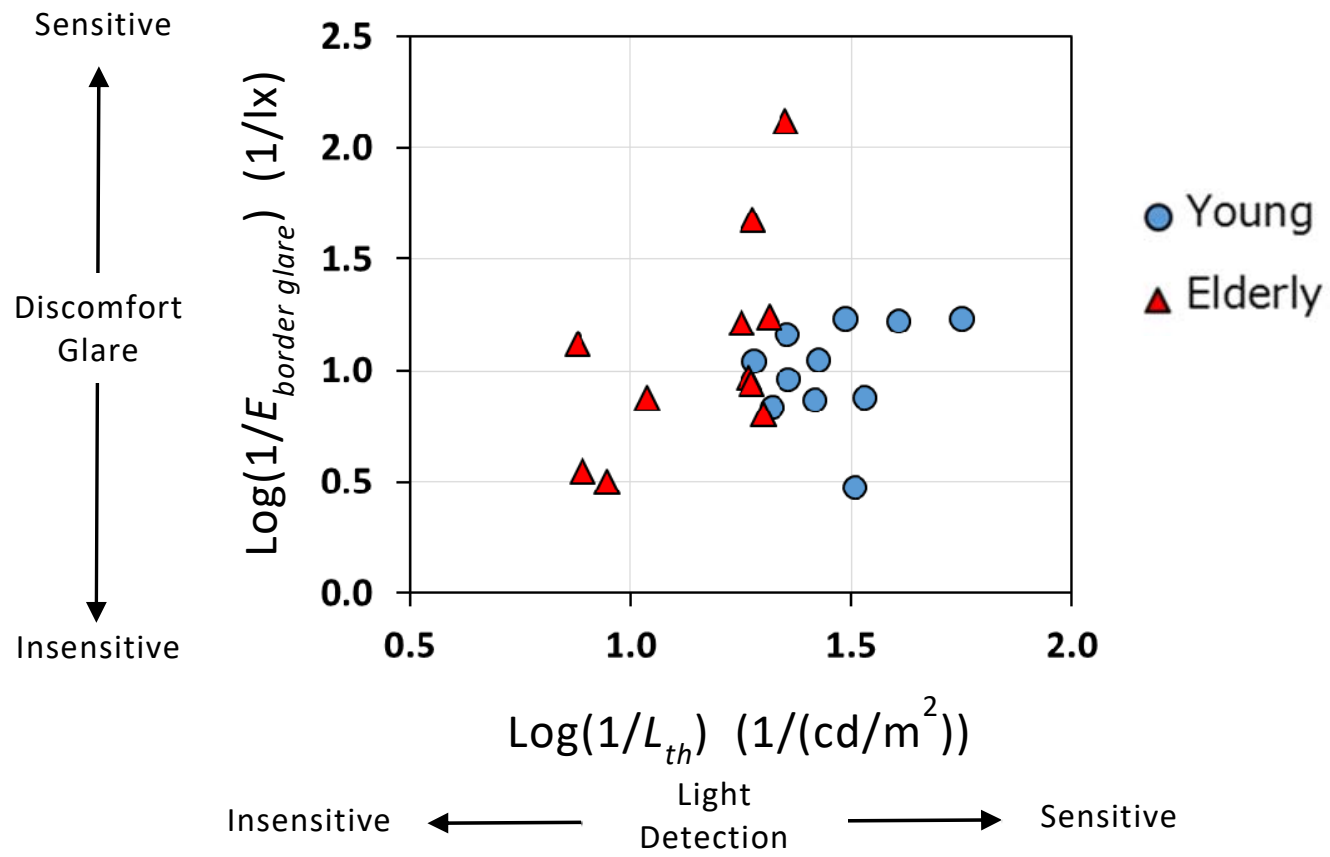

Figure 5 - Correlation between visual sensitivity and the sensitivity of discomfort glare

\subsection{Triad relation of intraocular straylight, visual sensitivity, and discomfort glare}

To see the mutual contribution of $\log s$ and $\log \left(1 / L_{t h}\right)$ to discomfort glare sensitivity, multiple regression analysis was applied to each of the young and elderly groups. In Figure 5, horizontal axis is the experimental values of log $\left(1 / L_{\text {border,glare }}\right)$, and vertical axis is the estimated values. Regression equations for the young and elderly groups are shown in equations (2) and (3), respectively below, 


$$
\begin{aligned}
& Y=0.31 \cdot X_{1}+0.11 \cdot X_{2}+5.08 \\
& Y=0.32 \cdot X_{1}+0.58 \cdot X_{2}-5.40
\end{aligned}
$$

where $Y, X_{1}$, and $X_{2}$, are standardized values of $\log \left(1 / L_{\text {border,glare }}\right), \log s$, and $\log \left(1 / L_{t h}\right)$, respectively. Fitting for the young observers is non-significant, implying that rating of discomfort glare is determined other factors for young observers. One possibility is psychological rating property of individual observers. On the other hand, fitting is relatively good for elderly observers showing correlation coefficient of 0.67 . Weighting coefficient of log $\left(1 / L_{t h}\right)$ is larger than that of $\log \mathrm{s}$, indicating stronger contribution of visual sensitivity than the intraocular straylight.

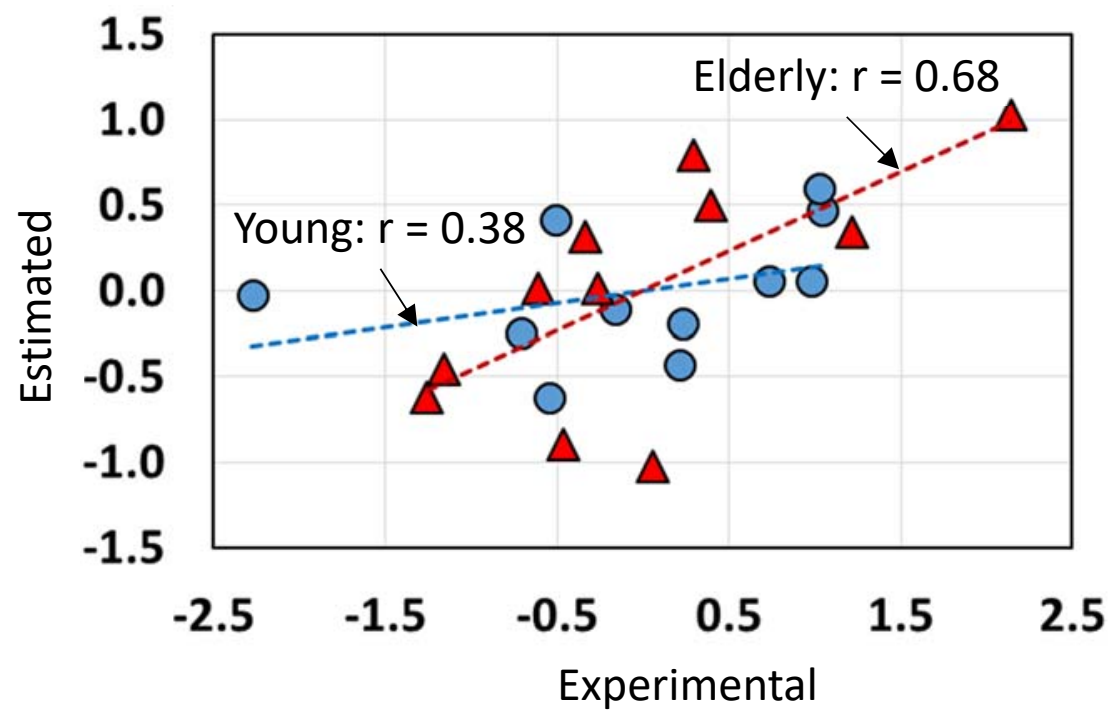

Figure 5 - Correlation between experimental and estimated values

\section{Conclusion}

Triad relation among 3 properties, intraocular straylight, visual sensitivity, and discomfort glare sensitivity, for elderly and young observers was investigated. Multiple regression analysis to estimate discomfort glare sensitivity using straylight parameter and visual sensitivity as explanatory variables was applied to the results of both groups. Correlation coefficient between the estimated and experimental values is 0.67 for elderly group, indicating that these two factors contribute to the subjective evaluation of discomfort glare for individual observers. On the other hand, estimation was not so good for young group, suggesting that other factors, e.g., psychological criterion difference of subjective rating, affect on individual differences of discomfort glare for young observers. Further investigation is needed to explore the causes of discomfort glare evaluation for young and elderly observers.

\section{References}

1) Vos, J. J. 2003. On the Cause of Disability Glare and its Dependence on Glare Angle, Age and Ocular Pigmentation, Clin. Exp. Optom., 86, 363-370.

2) Van den Berg, T. J. et al. 2010. Ocular Media Clarity and Straylight, Encyclopedia of Eye, 3, 173183.

3) Ayama, M. et al. 2015. Estimation of Straylight in the Eye and its Relation to Visual Function, Optical Review, 21, 185-196.

4) Tsuchiya, N. et al. 2017. Age and Wavelength Dependence of Intraocular straylight. Proceedings of the 13th AIC Congress 2017, USB. 
5) CIE 1983. CIE 055:1983. Discomfort Glare in the Interior Working Environment. Vienna: CIE.

6) Kasahara, T. et al. 2006. Discomfort Glare Caused by White LED Light Sources. J. Light \& Vis. Env., 30, 95-103.

7) Kimura-Minoda, K. and Ayama, M. 2010. Evaluation of Discomfort Glare from Color LEDs and its Correlation with Individual Variations in Brightness Sensitivity, Color Res. Appl., 36, 286-294.

8) Tashiro, T. et al. 2015. Discomfort Glare of White LED lights with Different Spatial Arrangement. Lighting Res. Technol., 47, 316-337.

9) Kohko, S. et al. 2015. Study on Evaluation of LED Lighting Glare in Pedestrian Zones, J. Light \& Vis. Env., 39, 15-25.

10) Yan Y. et al. 2017. Assessing Glare. Part 1: Comparing uniform and non-uniform LED luminaires, Lighting Res. Technol., 49, 195-210

11) Huang, W.J. et al. 2018. Discomfort Glare Caused by White LEDs having Different Spectral Power Distributions, Lighting Res. Technol., 50, 921-936.

12) Bargary, G. et al. 2015. Mechanism for Discomfort Glare in Central Vision, Invest Ophthal. Vis. Sci., $56,464-471$. 